\title{
AVALIAÇÃO ESPAÇO-TEMPORAL DA CLOROFILA $a$ E DO ÍNDICE DE ESTADO TRÓFICO NO RIO PIRACICABA, SÃO PAULO, BRASIL.
}

\author{
SPATIAL AND TEMPORAL ASSESSMENT OF \\ CHLOROPHYLL $a$ AND THE THROPHIC STATE INDEX \\ IN THE PIRACICABA RIVER SÃO PAULO, BRAZIL
}

\author{
Rafael Grossi Botelho; Valdemar Luiz Tornisielo
}

\author{
Centro de Energia Nuclear na Agricultura/Universidade de São Paulo. E-mail \\ rbotelho@cena.usp.br
}

\begin{abstract}
RESUMO
Uma avaliação espaço-temporal das concentrações de clorofila $a$ bem como a determinação do índice de estado trófico (IET) na água do rio Piracicaba foram realizados entre março de 2011 a janeiro de 2012. Amostras de água foram coletadas mensalmente ao longo do rio em seis pontos e analisadas. Durante o estudo, amostras de água coletadas em todos os locais de amostragens assim como em todos os meses apresentaram valores de clorofila $a$ abaixo do estabelecido pela legislação ambiental brasileira. A água coletada nos meses de outubro e novembro e aquelas amostradas à montante e à jusante de Piracicaba foram as que apresentaram maiores valores de IET baseado na clorofila $a$. Pelo fato do rio Piracicaba estar localizado em uma área altamente industrializada e habitada, esperava-se observar valores de IET mais elevados nos pontos amostrados. Deve-se ressaltar, no entanto, que a diluição de nutrientes oriundos das atividades agrícolas e industriais, além da inibição do crescimento das algas por parte de contaminantes presentes na água do rio Piracicaba, pode estar associada aos baixos valores não significando desta forma que este ambiente possua baixa produtividade e que não esteja eutrofizado.
\end{abstract}

Palavras-chave: Ambiente Aquático. Clorofila $a$. Índice de Estado Trófico.

\begin{abstract}
A spatiotemporal assessment of chlorophyll $a$ concentrations as well as the determination of trophic state index (TSI) was conducted in the Piracicaba River water from March 2011 to January 2012 through chlorophyll $a$ concentration. Water samples were collected monthly at six different locations and analyzed. During the study period, water samples collected in all sampling sites and in all months presented values of chlorophyll $a$ below the limit set by the Brazilian environmental law. Water collected in October and November showed higher TSI and those sampled downstream and upstream of Piracicaba presented the highest values of TSI, however, were not classified as eutrophic. Due to the Piracicaba River be located in a highly populated and industrialized area, value of TSI should be higher and more frequently in the samples. The dilution of the nutrients from agricultural and industrial activities located in the Piracicaba River area in addition to the inhibition of the algae growth by contaminants may be associated with the low values, and thus, does not mean that the Piracicaba River has a low productivity.
\end{abstract}

Keywords: Aquatic Environment. Chlorophyll $a$. Trophic State Index. 


\section{INTRODUÇÃO}

Ao longo das últimas décadas muitos ambientes de água doce têm sido poluídos. A eutrofização, que se refere ao enriquecimento por nutrientes (principalmente nitrogênio e fósforo) e matéria orgânica, tornou-se um problema de qualidade de água, generalizado pelas atividades antrópicas nas bacias hidrográficas (CLOERN, 2001).

Entre algumas das várias causas da eutrofização, o uso de fertilizantes na agricultura, e descarga de efluentes industriais e domésticos não tratados merecem destaques (BOESCH, 2002), no entanto, a entrada de nutrientes pode se dar naturalmente (WETZEL, 2001). Dentre suas consequências para o ambiente estão a perda de vegetação aquática submersa, depleção de oxigênio, alteração da composição e estrutura das comunidades, e perda de biodiversidade (GLIBERT et al., 2010).

Mundialmente, a água esteticamente aceitável é uma necessidade na sociedade moderna e seu comprometimento por meio da eutrofização pode causar efeitos econômicos negativos, em países desenvolvidos e em desenvolvimento (SMITH e SCHINDLER, 2009). Com o aumento da população ao longo dos anos, espera-se que a eutrofização dos ambientes aquáticos se intensifique nas próximas décadas devido, principalmente, às atividades antrópicas (ELSER e BENNETT, 2011). Desta forma, o acompanhamento e avaliação da qualidade da água para estes ambientes são necessários e assim será possível traçar planos de manejo e recuperação.

As algas são componentes presentes em abundância nas comunidades planctônicas e estão envolvidas nos fluxos de energia e matéria em todos os ecossistemas aquáticos, incluindo reservatórios, lagos, córregos, rios, pântanos e mares. Elas utilizam diretamente a energia da luz, consumem o dióxido de carbono, e produzem oxigênio (GRAHAM et al., 2009). Como são organismos unicelulares com uma rápida regeneração em um curto período de tempo, as algas respondem mais rapidamente do que qualquer outro organismo quando há alteração em termos de estrutura de uma comunidade. Desta forma, estes organismos têm sido utilizados para avaliar a qualidade da água e medir a eficácia da restauração, devido à sua posição única na base de cadeias alimentares aquáticas (RAKOCEVIC-NEDOVIC e HOLLERT, 2005).

Tais problemas para o ambiente aquático, associados à eutrofização fizeram com que este assunto fosse tratado com mais atenção (YU et al., 2011), e desta forma com o tempo muitos métodos foram sendo desenvolvidos para avaliar o estado trófico dos ambientes aquáticos (PAERL, 2009; PRIMPAS e KARYDIS, 2010; DEVLIN et al., 2011; FERREIRA et al., 2011). A partir dessas metodologias, vários estudos começaram a ser desenvolvidos com o objetivo de determinar o IET de ecossistemas aquáticos (ZANINI et al., 2010; YU et al., 2011; ALVES et al., 2012; YANG et al., 2012).

Carlson (1977) desenvolveu uma classificação do estado trófico dos ambientes aquáticos baseado em três tipos de parâmetros: concentração de clorofila $a$, transparência da água medida pelo disco de Secchi, e concentração de fósforo total. Apesar de alguns autores utilizarem todos os três parâmetros, ou apenas fósforo e clorofila $a$, e até mesmo somente clorofila a, segundo Carlson (1977) qualquer um deles podem ser utilizado sozinho para tal análise. Desta forma, o objetivo deste estudo foi de determinar o IET das águas do Rio Piracicaba (São Paulo, Brasil) utilizando a variável clorofila $a$.

\section{MATERIAIS E MÉTODOS}

\section{Caracterização da área de estudo}

O rio Piracicaba está localizado na região Sudeste do Brasil (Estado de São Paulo) e é formado à montante da cidade de Americana através da junção dos rios Atibaia e Jaguari e deságua 
na represa de Barra Bonita próximo à cidade de Santa Maria da Serra, percorrendo assim cerca de $250 \mathrm{~km}$. O uso da terra ocorre principalmente através do cultivo de pastagens, citros e cana-deaçúcar. A região do rio Piracicaba apresenta um crescimento populacional e industrial maior do que a média do país, e devido ao acelerado desenvolvimento tornou-se um polo de diversas atividades altamente consumidoras e degradadoras dos recursos hídricos. Vêm ocorrendo aumento do consumo de água e das cargas de esgotos urbanos e agroindustrial, mudanças no uso da terra, e estas são as principais causas da alteração tanto da quantidade quanto da qualidade dos recursos hídricos (KRUSH et al., 1997; MARTINELLI e VICTORIA, 1997).

O rio Piracicaba possui três pontos de monitoramento de pluviosidade sendo estes localizados na cidade de Americana, Piracicaba e Artemis. Durante o estudo, os dados pluviométricos para o rio Piracicaba foram adquiridos do Sistema de Alerta a Inundações do Estado de São Paulo.

\section{Período e locais de amostragem}

Amostras de água foram coletadas mensalmente no Rio Piracicaba durante os meses de março de 2011 a janeiro de 2012 em seis diferentes pontos ao longo do rio e foram assim determinados: Ponto 1 (montante de Americana - S $22^{\circ} 41.260^{\circ} \mathrm{WO} 47^{\circ} 18.678^{\circ}$ ), Ponto 2 (jusante de Americana - S $22^{\circ} 42.651^{\circ} \mathrm{WO} 47^{\circ} 25.708^{\circ}$ ), Ponto 3 (Montante de Piracicaba - S $22^{\circ} 41.347^{\circ}$ WO $47^{\circ} 35.003^{\circ}$ ), Ponto 4 (jusante de Piracicaba - S $22^{\circ} 41^{\prime} 748^{\prime \prime}$ WO $47^{\circ} 40.285^{\prime \prime}$ ), Ponto 5 (distrito de Artemis - S $22^{\circ} 41.580^{\circ} \mathrm{WO} 47^{\circ} 46.737^{\circ}$ ), Ponto 6 (Represa de Barra Bonita - S $22^{\circ} 37$. $666^{`}$ WO $48^{\circ} 10.430^{`}$ ). A figura 1 mostra a localização dos pontos de amostragem.

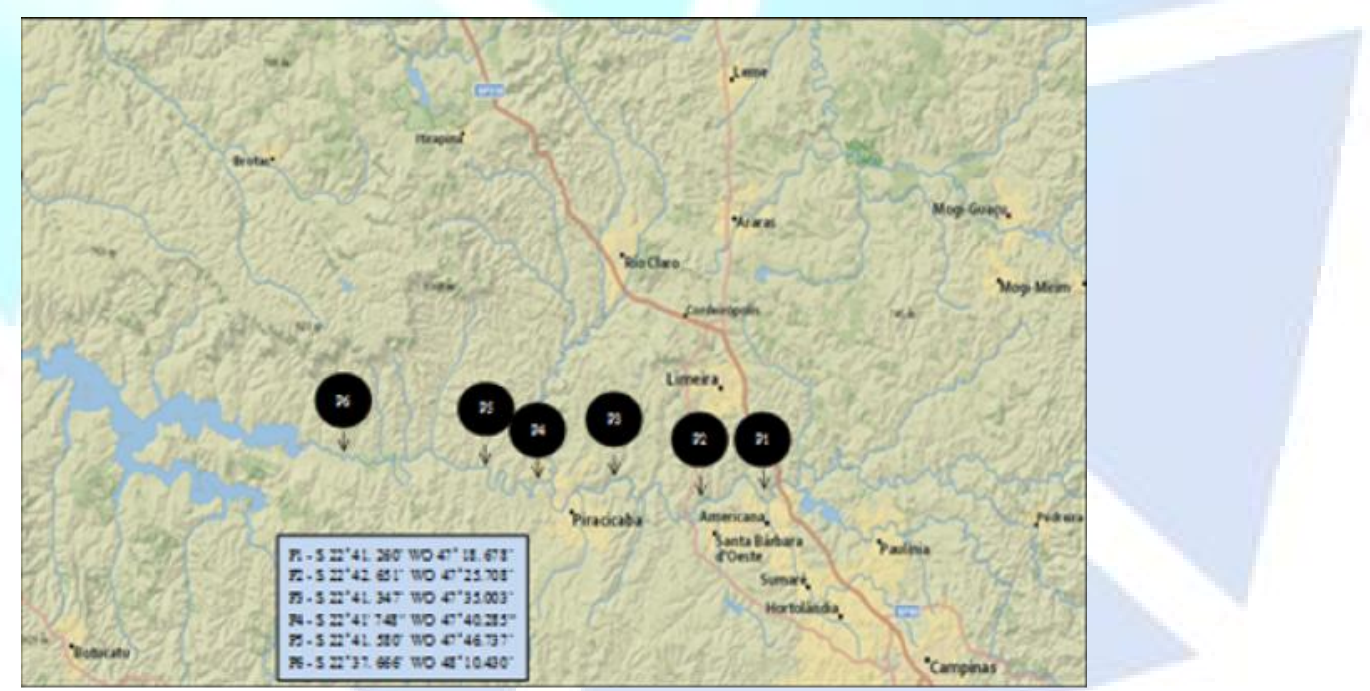

Figura 1. Localização dos pontos de amostragem no Rio Piracicaba. Fonte: Botelho et al. (2013).

As amostras foram armazenadas em frascos de vidro âmbar escuro e cobertas com plástico preto para evitar a exposição à luz. Logo após a coleta, as amostras foram levadas ao Laboratório de Ecotoxicologia do Centro de Energia Nuclear na Agricultura/Universidade de São Paulo para análise.

\section{Determinação de clorofila $a$ e IET}

Para avaliar o estado trófico das águas do rio Piracicaba foi utilizado o índice desenvolvido por Carlson (1997) para clorofila a e adaptado para águas dos rios do Estado de São Paulo por Lamparelli (2004). 

Tabela 1.

Os resultados do IET foram comparados utilizando-se a classificação de acordo com a

Tabela 1 - Classificação do Índice de Estado Trófico com a ponderação para Clorofila a segundo Lamparelli (2004).

\begin{tabular}{|c|c|c|}
\hline Categoria & Ponderação & Clorofila $a\left(\mu g \mathrm{~L}^{-1}\right)$ \\
\hline Ultraoligotrófico & $\mathrm{IET} \leq 47$ & $\mathrm{CL} \unlhd 0,74$ \\
\hline Oligotrófico & 47<IET 52 & $0,74 \leq \mathrm{CL} \leq 1,31$ \\
\hline Mesotrófico & $52<$ IET $\leq 59$ & $1,31 \leq \mathrm{CL} \leftrightharpoons 2,96$ \\
\hline Eutrófico & $59<$ IET $\leq 63$ & $2,96 \leq \mathrm{CL} \leq 4,70$ \\
\hline Supereutrófico & $63<$ IET $\leq 67$ & $4,70 \leq \mathrm{CL} \leq 7,46$ \\
\hline Hipereutrófico & IET $>67$ & $7,46<\mathrm{CL}$ \\
\hline
\end{tabular}

\section{RESULTADOS E DISCUSSÃO}

Durante este estudo, os meses que apresentaram alto índice pluviométrico foram março, abril e outubro de 2011 e janeiro de 2012 para Piracicaba, novembro e dezembro de 2011 e janeiro de 2012 para Americana, e outubro de 2011 para Artemis (Figura 2).

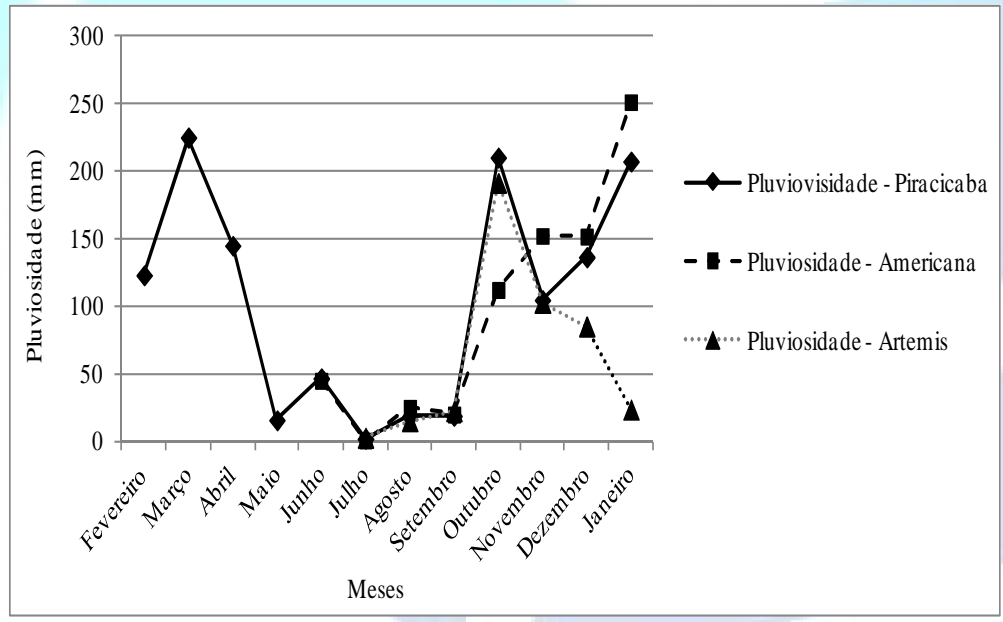

Figura 2. Pluviosidade (mm) do Rio Piracicaba durante o período do estudo.

Os valores das concentrações de clorofila $a$ encontrados nas amostras durante o período do estudo estão apresentados na Tabela 2.

O Conselho Nacional do Meio Ambiente (CONAMA) por meio da resolução 357 de 2005 (BRASIL, 2005). dispõe sobre a classificação e diretrizes ambientais para o enquadramento dos corpos de água superficiais, bem como condições e padrões de lançamento de efluentes.

Um dos parâmetros da água levado em consideração é a concentração de clorofila $a$. De acordo com o CONAMA, para água doce superficial de classe 1 (abastecimento para consumo humano após) tratamento simplificado, classe 2 (abastecimento para consumo humano após 
tratamento convencional), e classe 3 (abastecimento para consumo humano após tratamento avançado) a concentração máxima de clorofila $a$ permitida é de 10,30 , e $60 \mu \mathrm{g} \mathrm{L}^{-1}$, respectivamente (BRASIL, 2005). No presente trabalho, em nenhuma amostra de água coletada durante o período do estudo observou-se valores maiores ou próximos a $10 \mu \mathrm{g} \mathrm{L}^{-1}$. A variação espacial e temporal das concentrações de clorofila $a$ presentes nas amostras de água do rio Piracicaba estão apresentadas na Tabela 2.

Tabela 2 - Concentração $\left(\mu \mathrm{g} \mathrm{L}^{-1}\right)$ de clorofila $a$ determinada nas amostras de água coletas em diferentes pontos de amostragens do Rio Piracicaba de março de 2011 a janeiro de 2012.

\begin{tabular}{ccccccccccccc}
\hline \multirow{2}{*}{ Locais } & \multicolumn{10}{c}{ Meses } \\
\cline { 2 - 13 } & Mar & Abr & Mai & Jun & Jul & Ago & Set & Out & Nov & Dez & Jan & Média \\
\hline Ponto 1 & 0,06 & 0,16 & 0,28 & 0,28 & 0,06 & 0,34 & 0,33 & 3,14 & 0,28 & 0,22 & 0,05 & 0,47 \\
Ponto 2 & 0,06 & 0,06 & 0,22 & 0,06 & 0,06 & 0,79 & 0,39 & 1,68 & 0,50 & 0,34 & 0,05 & 0,38 \\
Ponto 3 & 0,06 & 0,16 & 0,16 & 0,11 & 0,06 & 0,17 & 0,61 & 1,63 & 1,46 & 0,78 & 0,05 & 0,47 \\
Ponto 4 & 0,06 & 0,06 & 0,22 & 0,06 & 0,06 & 0,28 & 0,56 & 2,86 & 1,40 & 0,90 & 0,05 & 0,59 \\
Ponto 5 & 0,06 & 0,06 & 0,16 & 0,11 & 0,06 & 0,28 & 0,89 & 1,63 & 1,01 & 0,28 & 0,05 & 0,42 \\
Ponto 6 & 0,06 & 0,28 & 0,06 & 0,05 & 0,11 & 4,1 & 0,06 & 0,06 & 0,22 & 0,05 & 0,05 & 0,46 \\
Média & 0,06 & 0,06 & 0,18 & 0,11 & 0,07 & 0,99 & 0,47 & 1,83 & 0,81 & 0,43 & 0,05 & \\
\hline
\end{tabular}

Durante o período de amostragem os locais que apresentaram maiores concentrações de clorofila $a$ baseado nos valores médios foram os ponto 4, 1, 3, 6 seguido pelos pontos 5 e 2 (Tabela 2). Segundo Ladim de Souza et al. (2009) maiores concentrações de clorofila $a$ tendem a ser encontradas em ambientes onde há grande disponibilidade de nutrientes inorgânicos dissolvidos, para produção primária oriunda principalmente de efluentes industriais. No caso do presente estudo, de todos os locais estudados aquele que sofreu menos a influência direta de efluentes industriais foi o ponto 6. Mesmo assim a disponibilidade de componentes inorgânicos oriundos das indústrias localizadas entre os pontos 1 e 5 podem ter contribuído com a eutrofização no ponto 6 , já que este local é represado (Represa de Barra Bonita) fazendo com que os nutrientes se concentrem neste ponto, conforme postulado no trabalho de Prado e Moraes Novo (2007).

Em relação à variação temporal de clorofila $a$ no rio Piracicaba, verificou-se que os meses que apresentaram maiores valores foram agosto, setembro, outubro e novembro de 2011. Os resultados para clorofila $a$ mostraram não estar relacionados com a precipitação durante o período de estudo na bacia do rio Piracicaba, já que em meses com maior precipitação como março, abril, dezembro e janeiro as concentrações de clorofila foram mais baixas se comparadas, por exemplo, com outubro onde também houve maior precipitação, porém com concentrações de clorofila elevadas (Tabela 2).

Sabe-se, no entanto, que o aumento da precipitação acarreta maior diluição de nutrientes (fósforo, nitrogênio e outros) no ambiente aquático, e isso pode ter influenciado nas baixas concentrações de clorofila $a$ no período chuvoso. A relação entre concentração de clorofila $a$ e precipitação não tem apresentado um padrão de acordo com alguns estudos. Bastos et al. (2005) encontraram maiores concentrações de clorofila $a$ em período chuvoso, ao contrário de Ladim de Souza et al. (2009) que encontraram valores mais altos em período seco. 
Segundo Smayda (1983), a caracterização da variabilidade de clorofila a longo prazo, como no presente estudo, é essencial para detectar efeitos antrópicos sobre a dinâmica do fitoplâncton. Além disso, entender a relação entre nutrientes e clorofila $a$ pode ajudar a evitar o processo de eutrofização.

Durante o período de amostragem o IET foi determinado em quatro categorias: ultraoligotrófico, oligotrófico, mesotrófico e eutrófico. Os locais de amostragem que apresentaram maiores valores médios de IET foram os pontos 3 e 4, seguido pelos pontos 1, 5, 2 e 6 (Tabela 3). Baseando-se nos valores médios todos os pontos se enquadraram dentro da classificação ultraoligotrófica (Tabela 4). Estes resultados mostraram que apesar de haver grande disponibilidade de nutrientes oriundos das atividades agrícolas, a produtividade pode estar sendo mascarada pela diluição destas substâncias já que o rio Piracicaba é extenso e possui um grande volume de água. Além disso, outro fator importante a ser considerado é a presença de poluentes tais como agrotóxicos, corantes têxteis e metais que podem estar inibindo o crescimento das algas.

Os dados apresentados para a avaliação temporal mostraram que de março a julho e dezembro e janeiro houve menor valor médio de IET (Tabela 3), e desta forma as amostras de água coletadas nestes meses foram classificadas como ultraoligotrófica (Tabela 4). Já as amostras coletadas entre os meses de agosto a novembro, apresentaram maiores valores de IET (Tabela 3), no entanto, mesmo assim foram classificadas como ultraoligotrófica, exceto no mês de outubro que se apresentou como oligotrófica (Tabela 4).

Os resultados apresentados de IET do rio Piracicaba para o período de estudo também não estão relacionados com a precipitação na bacia, pois em meses como agosto e setembro que teve baixas precipitações obtiveram valores altos de IET, enquanto que outubro e novembro apresentaram alta pluviosidade e altos valores de IET.

A Companhia de Tecnologia e Saneamento Ambiental (CETESB) do Estado de São Paulo realiza anualmente uma avaliação da qualidade de águas superficiais das principais Bacias Hidrográficas do estado, incluindo a Bacia do Rio Piracicaba. Para avaliação do Índice de Estado Trófico, a CETESB leva em consideração tanto clorofila $a$ como fósforo. No último relatório divulgado pela CETESB (CETESB, 2012) referente ao ano de 2012 os valores de clorofila variaram de 6,4 a 7,7 $\mu \mathrm{g} . \mathrm{L}^{-1}$ com media de $6,84 \mu \mathrm{g} . \mathrm{L}^{-1}$, sendo abaixo do limite estabelecido pelo CONAMA, assim como no presente trabalho. Valores de IET variaram de 56 a $60 \mathrm{com}$ valor médio de 58,5, o que o caracterizou como mesotrófico.

Tabela 3 - Índice de Estado Trófico (IET) determinado nas amostras de água coletas em diferentes pontos de amostragens do Rio Piracicaba de março de 2011 a janeiro de 2012.

\begin{tabular}{lcccccccccccc}
\hline \multirow{2}{*}{ Locais } & \multicolumn{10}{c}{ Meses } \\
\cline { 2 - 14 } & Mar & Abr & Mai & Jun & Jul & Ago & Set & Out & Nov & Dez & Jan & Média \\
\hline Ponto 1 & 25,10 & 34,23 & 39,10 & 39,10 & 25,10 & 40,70 & 40,50 & 60,00 & 39,10 & 37,01 & 25,10 & 36,82 \\
Ponto 2 & 25,10 & 25,10 & 37,01 & 25,10 & 25,10 & 48,05 & 41,90 & 54,60 & 44,14 & 40,50 & 25,10 & 35,60 \\
Ponto 3 & 25,10 & 34,23 & 34,23 & 31,00 & 25,10 & 34,25 & 45,80 & 54,30 & 53,30 & 49,20 & 25,10 & 37,41 \\
Ponto 4 & 25,10 & 25,10 & 37,01 & 25,10 & 25,10 & 39,10 & 45,10 & 59,30 & 53,00 & 48,60 & 25,10 & 37,05 \\
Ponto 5 & 25,10 & 25,10 & 34,23 & 31,00 & 25,10 & 39,10 & 48,60 & 54,30 & 50,20 & 39,10 & 25,10 & 36,08 \\
Ponto 6 & 25,10 & 39,10 & 25,10 & 25,10 & 31,00 & 62,40 & 25,10 & 25,10 & 37,01 & 25,10 & 25,10 & 31,38 \\
Média & 25,10 & 28,14 & 34,44 & 29,40 & 26,08 & 43,93 & 41,16 & 51,26 & 46,12 & 39,91 & 25,10 & \\
\hline
\end{tabular}


Tabela 4 - Classificação do Índice de Estado Trófico (IET) das amostras de água coletadas em diferentes pontos de amostragens no Rio Piracicaba de março de 2011 a janeiro de 2012.

\begin{tabular}{cccccccccccccc}
\hline & \multicolumn{10}{c}{ Meses } \\
\hline Locais & Mar & Abr & Mai & Jun & Jul & Ago & Set & Out & Nov & Dez & Jan & $\begin{array}{c}\text { Classificação } \\
\text { geral }\end{array}$ \\
Ponto 1 & Ultra & Ultra & Ultra & Ultra & Ultra & Ultra & Ultra & Eutro & Ultra & Ultra & Ultra & Ultra \\
Ponto 2 & Ultra & Ultra & Ultra & Ultra & Ultra & Oligo & Ultra & Meso & Oligo & Ultra & Ultra & Ultra \\
Ponto 3 & Ultra & Ultra & Ultra & Ultra & Ultra & Ultra & Ultra & Meso & Meso & Oligo & Ultra & Ultra \\
Ponto 4 & Ultra & Ultra & Ultra & Ultra & Ultra & Ultra & Ultra & Meso & Meso & Oligo & Ultra & Ultra \\
Ponto 5 & Ultra & Ultra & Ultra & Ultra & Ultra & Ultra & Oligo & Meso & Meso & Ultra & Ultra & Ultra \\
$\begin{array}{c}\text { Ponto 6 } \\
\begin{array}{c}\text { Classificação } \\
\text { geral }\end{array}\end{array}$ & Ultra & Ultra & Ultra & Ultra & Ultra & Eutro & Ultra & Ultra & Ultra & Ultra & Ultra & Ultra \\
\hline
\end{tabular}

Os resultados apresentados pela CETESB sugerem que o rio Piracicaba ao longo do tempo pode vir a apresentar eutrofização já que no presente estudo (realizado em março de 2011 a janeiro de 2012) o mesmo foi caracterizado como ultraoligotrófico e no da CETESB como mesotrófico. Existe uma diferença entre os dois estudos. No presente trabalho, utilizou-se somente o parâmetro clorofila $a$ diferentemente da CETESB, e desta forma os resultados da CETESB podem ter apresentados maiores valores de IET devido à avaliação em conjunto com o fósforo total.

A relação entre fósforo e clorofila $a$ na determinação do IET, no entanto, não tem seguido um padrão, como demonstrou Maranho (2011). Nesse estudo, o IET foi consideravelmente maior baseado na concentração de clorofila $a$ comparado quando se utilizou o fósforo. Pelo fato da determinação de baixas concentrações de fósforo, quando o IET foi avaliado por meio de dois parâmetros, o rio Corumbataí apresentou-se como ultraoligotrófico na maior parte do período amostrado e na maioria dos pontos.

Avaliando a concentração de clorofila $a$ no litoral de Santa Catarina (Brasil), Proença (2002) encontrou concentrações variando entre 0,19 e $10,53 \mu \mathrm{g} \mathrm{L}^{-1}$. Silvério da Silva et al. (2010) observaram valores superiores aos determinados no presente estudo, inclusive maiores que o permitido na legislação brasileira para o Rio São Francisco Falso, localizado no estado do Paraná. Em outro estudo, Souza et al. (2009) encontraram valores próximos de $60 \mu \mathrm{g} \mathrm{L}^{-1}$ para o estuário do Rio Cachoeira (Nordeste do Brasil). No caso destes estudos citados anteriormente, os altos valores de clorofila $a$ se devem a influencias de indústrias e a precipitação antes do período de amostragem. Estudos realizados fora do Brasil também merecem destaques. Thiemann e Kaufmann (2000) encontraram valores variando entre 1 a $90 \mu \mathrm{g} \mathrm{L}^{-1}$ para um lago na Alemanha. Já Yang et al. (2012), encontraram valores ainda maiores, variando entre $17.05 \pm 1.29$ e $264.54 \pm 15.37 \mu \mathrm{g} \mathrm{L}^{-1}$.

Thiemann e Kaufmann (2000) avaliando o estado trófico de cinco lagos na Alemanha encontraram valores de IET variando entre 40 a 75. Em outro estudo, Yang et al. (2012) encontraram IET de 85,29 como o valor mais alto dentre os onze reservatórios estudados. Estes valores foram bem maiores se comparados aos do presente estudo, onde o menor valor encontrado foi de 25,10 e o máximo de 62,40. Em estudo realizado no Brasil, Affonso et al. (2011) encontraram valores extremamente altos para clorofila $a$, e por conta disso o Lago Grande de Curuaí (Pará) onde foi realizado o estudo obteve valores altos de IET. Em todos os três trabalhos, foi utilizado o parâmetro clorofila a para cálculo do IET, porém esses estudos ocorreram em lagos ou reservatórios, ou seja, ambientes lênticos, diferentemente do presente trabalho que ocorreu em um ambiente lótico onde a vazão e extensão pode ser um fator determinante para a determinação de 
baixos valores. Além disso, em ambientes lóticos o efeito da diluição pode ser um fator importante na determinação de baixos valores de clorofila $a$ e IET.

Vários estudos já abordaram problemas da eutrofização em ambientes aquáticos prejudicando a necessidade e manutenção tanto dos seres humanos como da biota aquática (ESPÍNDOLA et al., 2000; SAMPAIO et al., 2002; SOTERO-SANTOS et al., 2008; GARCIA et al. 2010).

\section{CONCLUSÃO}

O presente estudo avaliou a distribuição espacial e temporal da clorofila $a$ nas águas do rio Piracicaba para posteriormente determinar o IET. Os resultados mostraram baixas concentrações de clorofila $a$ nas amostras de água o que levou a determinação de baixos valores de IET. Considerase, porém, que tanto o volume de água deste rio e à inibição do crescimento de algas por parte de contaminantes presentes neste ambiente podem ter mascarado os resultados, e desta forma, não significando que o rio Piracicaba não esteja eutrofizado. Sendo assim, recomendamos que na avaliação de IET para rios que sofrem grandes influencias industrial e agrícola como o rio Piracicaba, torna-se importante determinar, além da clorofila $a$ nas amostras de água, concentrações de fósforo e nitrogênio que são nutrientes associados ao fenômeno de eutrofização. Além disso, a avaliação do IET no rio Piracicaba deve ser periodicamente realizada, já que a região onde o rio está localizado apresenta características que contribuem para este processo. Destaca-se ainda a importância da inclusão da determinação do IET em estudos de monitoramento de ambientes aquáticos já que esta é uma importante ferramenta na determinação da eutrofização.

\section{AGRADECIMENTOS}

À Fundação de Amparo à Pesquisa do Estado de São Paulo pelo financiamento (2010/04756-4 e 2010/18895-6).

\section{REFERÊNCIAS}

AFFONSO, A.G.; BARBOSA, C.; NOVO, E.M.L.M. Water quality changes in floodplain lakes due to the Amazon River flood pulse: Lago Grande de Curuaí (Pará). Brazilian Journal of Biology, São Carlos, v.71, n.3, p.601-610, 2010.

ALVES, I.C.C.; EL-ROBRINI, M.; SANTOS, M.L.S.; MONTEIRO, S.M.; BARBOSA, L.P.F.; GUIMARÃES, J.T.F. Qualidade das águas superficiais e avaliação do estado trófico do Rio Arari (Ilha de Marajó, norte do Brasil). Acta Amazonica, Manaus, v.42, n.1, p.115 - 124, 2012.

BASTOS, R.B.; FEITOSA, F.A.N.; MUNIZ, K. Variabilidade espaço-temporal da biomassa fitoplanctônica e hidrologia no estuário do Rio Uma (Pernambuco - Brasil). Tropical Oceanography, Pernambuco, v.3, n.1, p.1-18, 2005.

BOESCH, D. Challenges and opportunities for science in reducing nutrient over-enrichment of coastal ecosystems. Estuaries and Coasts, Port Republic, v.25, n.4, p.744-758, 2002. 
BOTELHO, R.G.; ROSSI, M.L.; MARANHO, L.A.; OLINDA, R.A.; TORNISIELO, V.L. Evaluation of Surface Water Quality using an Ecotoxicological Approach: A Case Study of the Piracicaba River (São Paulo, Brazil). Environmental Science and Pollution Research International, Landsberg, v.20, n.7, p.4382-4395, 2013.

BRASIL. Ministério do Meio Ambiente. Conselho Nacional do Meio Ambiente. Resolução n.357, de 17 de março de 2005. Dispõe sobre a classificação dos corpos de água e diretrizes ambientais para o seu enquadramento, bem como estabelece as condições e padrões de lançamento de efluentes, e dá outras providências. Brasília: CONAMA, 2005. 27p. Disponível em: http://www.mma.gov.br/port/conama/res/res05/res35705.pdf Acesso em: 09 Nov. 2013.

CARLSON, R.E. A trophic state index for lakes. Limnology and Oceanography, Waco, v.22, n.2, p.361-369, 1977.

CLOERN, J.E. Our evolving conceptual model of the coastal eutrophication problem. Marine Ecology Progress Series, Oldendorf/Luhe, v.210, p. 223-253, 2001.

CETESB. 2012. Companhia de Tecnologia de Saneamento Ambiental. Qualidade das águas superficiais no Estado de São Paulo. São Paulo: CETESB, 370p.

DEVLIN, M.; BRICKER, S.; PAINTING, S. Comparison of five methods for assessing impacts of nutrient enrichment using estuarine case studies. Biogeochemistry, Netherlands, v.106, n.2, p.177$205,2011$.

ELSER, J.J.; BENNETT, E. Phosphorus cycle: A broken biogeochemical cycle. Nature, London, v.478, n.7367, p.29-31, 2011.

ESPÍNDOLA, E.L.G.; MATSUMURA-TUNDISI, T.; RIETZLER, A.C.; TUNDISI, J.G. Spatial heterogeneity of the Tucuruí reservoir (State of Pará, Amazonia, Brazil) and the distribution of zooplanktonic species. Brazilian Journal of Biology, São Carlos, v. 6, n.2, p.179-194, 2000.

FERREIRA, J.G.; ANDERSEN, J.H.; BORJA, A.; BRICKER, S.; CAMP, J.; CARDOSO DA SILVA, M.; GARCÉS, E.; HEISKKANEN, A.S.; HUMBORG, C.; IGNATIADES, L.; LANCELOT, C.; MENESGUEN, A.; TETT, P.; HOEPFFNER, N.; CLAUSSEN, U. Overview of eutrophication indicators to assess environmental status within the European Marine Strategy Framework Directive. Estuarine, Coastal and Shelf Science, London, v.93, n.2, p.117-131, 2011.

GARCIA, A.C.; BARGU, S.; DASH, P.; RABALAIS, N.; SUTOR, M.; MORRISON, W.; WALKER, N.D. Evaluating the potential risk of microcystins to blue crab (Callinectes sapidus) fisheries and human health in a eutrophic estuary. Harmful Algae, Amsterdam, v.9, n.2, p.134-143, 2010.

GLIBERT, P.M.; ALLEN, J.I.; BOUWMAN, A. F.; BROWN, C. W.; FLYNN, K. J.; LEWITUS, A.J.; MADDEN, C.J. Modeling of HABs and eutrophication: status, advances, challenges. Journal of Marine Systems, Amsterdam, v.83, n.3-4, p.262-275, 2010.

GRAHAM LE, GRAHAM JM, WILCOX LW. Algae. 2nd ed. Benjamin Cumming, New York. 2009, 616p. 
KRUSCH, A.V.; CARVALHO, F.P.; MORAES, J.M.; CAMARGO, P.B.; BALLESTER, M.V. R.; HORNINK, S.; MARTINELLI, L.A.; VICTORIA, R.L. Spatial and temporal water quality variability in the Piracicaba River Basin, Brazil. Journal of the Americans Water Resources Association, Middleburg, v.33, n.5, p.1117-1123, 1997.

LADIM DE SOUSA, M.F.; EÇA, G.F.; SILVA, M.A.M.; AMORIM, F.A.C.; LOBO, I.P. Distribuição de nutrientes dissolvidos e clorofila $a$ no estuário do Rio Cachoeira, Nordeste do Brasil. Atlântica, Rio Grande, v.31, n.1, p.107-121, 2009.

LAMPARELLI, M.C. Grau de trofia em corpos d'água do estado de São Paulo: avaliação dos métodos de monitoramento. 2004. 235f. Tese (Doutorado em Ciências) - Universidade de São Paulo, 2004.

MARANHO, L.A. Avaliação da qualidade da água do rio Corumbataí (SP) por meio de variáveis bióticas e abióticas. 2012. 106f. Tese (Doutorado em Ciências) - Centro de Energia Nuclear na Agricultura, Universidade de São Paulo, 2012.

MARTINELLI, L.A. VICTORIA, R.L. Spatial and temporal water quality variability in the Piracicaba River Basin, Brazil. Journal of American Water Resources Association, Middleburg, v.33, n.5, p.1117-1123, 1997.

PAERL, H.W. Controlling eutrophication along the freshwater-marine continuum: dual nutrient (N and P) reductions are essential. Estuaries and Coasts, Port Republic, v.32, n.4, p.593-601, 2009.

PRIMPAS, I.; KARYDIS, M. Improving statistical distinctness in assessing trophic levels: the development of simulated normal distributions. Environmental Monitoring and Assessment, Dordrecht, v.169, n.1-4, p.353-365, 2010.

PRADO, R.B.; MORAES NOVO, E.M.L. Avaliação espaço-temporal da relação do estado trófico do Reservatório de Barra Bonita (SP) com o potencial poluidor da Bacia Hidrográfica. Sociedade e Natureza, Uberlândia, v.19, n.2, p.5-18, 2007.

PROENÇA, L.A.O. Clorofila $a$ do fitoplâncton em seis enseadas utilizadas para o cultivo de moluscos bivalves no litoral de Santa Catarina. Notas Técnicas Facimar, Itajaí, v.6, n.1, p.33-44, 2002.

RAKOCEVIC-NEDOVIC J.; HOLLERT H. Phytoplankton community and chlorophyll $a$ as trophic state indices of Lake Skadar (Montenegro, Balkan). Environmental Science and Pollution Research International, Landsberg, v.12, n.3, p.146-152, 2005.

SAMPAIO, E.V.; ROCHA, O.; MATSUMURA-TUNDISI, T.; TUNDISI, J.G. Composition and abundance of zooplankton in the limnetic zone of seven reservoirs of the Paranapanema River. Brazilian Journal of Biology, São Carlos, v.62, n.3, p.525-545, 2002.

SILVÉRIO DA SILVA, G.; MIOLA, S.; SILVÉRIO DA SILVA, G.; SOUZA, E.R. Avaliação das águas do Rio São Francisco Falso, Tributário do Reservatório de Itaipú, Paraná. Eclética Química, Araraquara, v.35, n.3, p. 117-122, 2010. 
SMAYDA, T.J. The plankton of estuaries. In: KETCHUM, B. H. (Ed.). Estuaries and enclosed seas. Amsterdam: Elsevier, 1983, p. 65-112.

SMITH, V.H.; SCHINDLER, D.W. Eutrophication science: where do we go from here? Trends in Ecology and Evolution, Amsterdam, v.24, n.4, p.201-207, 2009.

SOTERO-SANTOS, R.B.; CARVALHO, E.G.; DELLAMANO-OLIVEIRA, M.J.; ROCHA, O. Occurrence and toxicity of an Anabaena Bloom in a tropical reservoir (Southeast Brazil). Harmful Algae, Amsterdam, v.7, n.5, p.590-598, 2008.

SOUZA, M.F.L.; EÇA, G.F.; SILVA, M.A.M.; AMORIN, F.A.C.; LÔBO, I.P. Distribuição de nutrientes dissolvidos e Clorofila-A no estuário do Rio Cachoeira, Nordeste do Brasil. Atlantica, Rio Grande, v.31, n.1, p.107-121, 2009.

THIEMANN, S.; KAUFMANN, H. Determination of Chlorophyll Content and Trophic State of Lakes Using Field Spectrometer and IRS-1C Satellite Data in the Mecklenburg Lake District, Germany. Remote Sensing of Environment, New York, v.73, n.2, p.227-235, 2000.

ZANINI, H.H.T.; AMARAL, L.A.; ZANINI, J.R.; TAVARES, L.H.S. Caracterização da água da microbacia do Córrego Rico avaliada pelo índice de qualidade de água e de estado trófico.

Engenharia Agrícola, Jaboticabal, v. 30, n.4, p.732-741, 2010.

WETZEL, RG. Limnology: lake and river ecosystems. Elsevier Academic Press, 2001. 1006 p.

YANG, J.; YU, X.; LIU, L.; ZHANG, W.; GUO, P. Algae community and trophic state of subtropical reservoirs in southeast Fujian, China. Environmental Science and Pollution Research International, Landsberg, v.19, n.5, p.1432-1442, 2012.

YU, H.B.; XI, B.D.; JIANG, J.Y.; HEAPHY, M.J.; WANG, H.L; LI, D.L. Environmental heterogeneity analysis, assessment of trophic state and source identification in Chaohu Lake, China. Environmental Science and Pollution Research International, Landsberg, v.18, n.8, p.13331342, 2011. 